\title{
Prediction Method for Time Series of Imagery Data in Eigen Space
}

\author{
Validity of the Proposed Prediction Metyhod for Remote Sensing Satellite Imagery Data
}

\author{
Kohei Arai \\ Graduate School of Science and Engineering \\ Saga University \\ Saga City, Japan
}

\begin{abstract}
Prediction method for time series of imagery data on eigen space is proposed. Although the conventional prediction method is defined on the real world space and time domains, the proposed method is defined on eigen space. Prediction accuracy of the proposed method is supposed to be superior to the conventional methods. Through experiments with time series of satellite imagery data, validity of the proposed method is confirmed.
\end{abstract}

Keywords-prediction method; eigen value decomposition; eigen space; time series analysis.

\section{INTRODUCTION}

There are conventional prediction methods which allow prediction of future imagery data by using the acquired imagery data in the past [1]-[6]. The conventional prediction method is defined on real world space and time domains, in particular, is defined as an auto regressive model. Namely, the conventional prediction model is using relations among the time series of data which is described with stochastic processes such as Markov model ${ }^{1}$. Therefore, prediction accuracy is not good enough when the time series of data has an irregularity in time domain.

On the other hands, it is possible to create eigen space [6] with time series of data. Namely, time series of data can be projected onto eigen space from the real world space and time domains. Time series of data behavior can be well described on the eigen space rather than the real world space and time domains in particular for the irregularly changed data. The conventional prediction models are based on autoregressive model, or moving average model. Therefore, it is difficult to predict a future data using previously acquired time series of data for irregularly varied data. Meanwhile, it is expected that prediction accuracy of the proposed method is better than that of the conventional method.

Through experiments with GMS/VISSR images ${ }^{2}$ which are acquired every one hour, comparative study on prediction accuracy between the proposed and the conventional methods is conducted. The experimental results show advantage of the proposed method in terms of prediction accuracy.

\footnotetext{
${ }^{1}$ http://en.wikipedia.org/wiki/Markov_model

${ }^{2}$ http://www.tric.u-tokai.ac.jp/tsic/egms.html
}

The following section describes the proposed prediction method for time series analysis followed by experimental results. Then conclusion is described together with some discussions.

\section{PRoposed Method}

\section{A. Data Description on Eigen Spece}

Eigen space is formed with the bases of eigen vectors determined in conjunction with eigen values of variancecovariance matrix derived from the vectors of acquired imagery data as vectors. The vectors are projected in the eigen space, then time series of imagery data can be represented as the vector in the eigen space.

$\mathbf{M}$ of imagery data, $\left\{\mathrm{f}^{i} \mid i=0, \cdots, M\right\}$, then imagery data matrix, $\mathbf{F}$ is defined as the following equation,

$$
\mathbf{F}=\left[\mathrm{f}^{1}-\mathbf{c}, \cdots, \mathrm{f}^{M}-\mathbf{c}\right]
$$

where the time series of imagery data,

$$
\mathbf{f}=\left[f_{1}, f_{2}, \cdots, f_{N}\right]^{T}
$$

in concern and the averaged images,

$$
\mathbf{c}=\sum_{i=1}^{M} \mathrm{f}^{i} \text {. }
$$

Then variance-covariance matrix is defined as follows,

$$
\mathbf{Q}=\mathrm{FF}^{T} \quad(N \times N)
$$

and eigen values of $\mathrm{Q}$ is expressed as follows,

$\lambda_{i} \mathbf{e}_{i}=\mathbf{Q} \mathbf{e}_{i}$

where $e_{i}$ is defined as follows,

$$
\begin{aligned}
& \mathbf{E}=\left[\mathbf{e}_{1}, \cdots, \mathbf{e}_{N}\right] \\
& \mathbf{e}_{i}=\left[e_{i 1}, e_{i 2}, \cdots, e_{i N}\right]^{T}
\end{aligned}
$$

The well known contribution factor is defined as follows,

$$
W_{k}=\frac{\sum_{i=1}^{k} \lambda_{i}}{\sum_{i=1}^{N} \lambda_{i}} \geq T_{s}
$$


Then $\mathrm{F}$ can be expressed with the first $\mathrm{k}$ eigen vectors,

$$
\hat{\mathbf{E}}=\left[\mathbf{e}_{1}, \cdots, \mathbf{e}_{k}\right]
$$

By using equation (4), time series of imagery data can be projected onto eugen space based on the following equation,

$$
\boldsymbol{\Phi}^{i}=\hat{\mathbf{E}}^{T}\left(\mathbf{f}^{i}-\mathbf{c}\right)
$$

That is same thing for representation of whole time series of imagery data by using all of the eigen vectors as follows,

$$
\begin{aligned}
& \hat{\boldsymbol{\Phi}}^{i}=\mathbf{E}^{T}\left(\mathbf{f}^{i}-\mathbf{c}\right) \\
& \hat{\boldsymbol{\Phi}}^{i}=\left[\phi_{1}^{i}, \cdots, \phi_{N}^{i}\right]
\end{aligned}
$$

The prediction method proposed here is based on auto regressive model with the projected time series of imagery data onto eigen space.

\section{B. Auto Regressive Model}

By using the following $\mathrm{n}-1$ of time series of data,

$$
\{x(s) \mid s=1,2, \cdots, n-1\}
$$

n-th data can be predicted as follows,

$$
\hat{x}(s)=\sum_{m=1}^{M} a(m) x(s-m)
$$

where

$$
\epsilon(s)=x(s)-\hat{x}(s) \quad(s=1,2, \cdots, M)
$$

is called prediction error. All the coefficients for equation (7) can be determined by minimizing the following mean square error,

$$
\lim _{N \rightarrow \infty} \frac{1}{N} \sum_{s=1}^{N} \epsilon^{2}(s)
$$

Thus autoregressive model can be represented as follows,

$$
x(s)=\sum_{m=1}^{M} a(m) x(s-m)+\epsilon(s) \quad(s=1,2, \cdots)
$$

Stationery condition can be expressed as follows,

$$
1-a(1) z-a(2) z^{2}-\cdots-a(M) z^{M}=0
$$

It is called characteristic equation of autoregressive model.

For the prediction error, the following three equations are well known,

$$
\begin{aligned}
& E[\epsilon(s) x(s)]=E\left[\epsilon^{2}(s)\right]=\sigma^{2} \\
& E\left[x^{2}(s)\right]=\sum_{m=1}^{M} a(m) E[x(s-m) x(s)]+\sigma^{2} \\
& E[x(s) x(s-k)]=\sum_{m=1}^{M} a(m) E[x(s-m) x(s-k)]
\end{aligned}
$$

Together with the following three autocorrelation functions,

$$
\begin{array}{ll}
R_{x x}(0)=\sum_{m=1}^{M} a(m) R_{x x}(m)+\sigma^{2} & \\
R_{x x}(k)=\sum_{m=1}^{M} a(m) R_{x x}(|k-m|) & (k=1,2, \cdots) \\
R_{x x}(k)=\sum_{m-1}^{M} a(m) R_{x x}(k-m) & (k \geq M)
\end{array}
$$

As of $R_{x x}(-k)=R_{x x}(k)$, then

$R_{x x}(0)=\sum_{m-1}^{M} a(m) R_{x x}+\sigma^{2}$

$R_{x x}(k)=a(k) R_{x x}(0)+\sum_{j-1}^{M}(a(k+j)+a(k-j)) R_{x x}(j)$

Therefore, if $\alpha^{\mathrm{N}}=\left(\alpha(\mathrm{I})^{2} \propto(\delta)^{2} \cdots^{2} \propto(\mathrm{W})\right)_{\mathrm{L} \text { and }} \sigma^{2}$ are given, then probability density function is reduced as follows,

$$
f\left(x \mid \sigma^{2}, a_{M}\right)=h\left(x_{M} \mid \sigma^{2}, a_{M}\right) \prod_{s=M+1}^{N} g\left(x(s) \mid \sigma^{2}, a_{M}\right)
$$

In order to determine the parameters in the probability density function,

$$
\begin{aligned}
\log f\left(x \mid \sigma^{2}, a_{M}\right) \\
=\log h\left(x_{M} \mid \sigma^{2}, a_{M}\right)+\sum_{s=M+1}^{N} \log g\left(x(s) \mid \sigma^{2}, a_{M}\right) \\
=\log h\left(x_{M} \mid \sigma^{2}, a_{M}\right)+(N-M) \log \left(2 \pi \sigma^{2}\right)^{-\frac{1}{2}} \\
\quad-\frac{1}{2 \sigma^{2}} \sum_{s=M+1}^{N}\left\{x(s)-\sum_{m=1}^{M} a(m) x(s-m)\right\}^{2}
\end{aligned}
$$

can be used. By maximizing the logarithmic function of the probability density function, then the parameters can be determined. It, however, is time consumable. Therefore the following Yule Walker method or the following least square method is used to be used.

\section{Least Square Method}

If $\mathrm{N}$ is much greater than $\mathrm{M}$, then the first term of the equation (9) is negligible. Thus, coefficients of the autoregressive model of equation (10) can be determined.

$$
\sum_{s=M+1}^{N}\left\{x(s)-\sum_{m=1}^{M} a(m) x(s-m)\right\}^{2}
$$

$\sigma^{2}$ can be determined with maximum likelihood manner,

$$
\frac{N-M}{2 \sigma^{2}}=\frac{1}{2\left(\sigma^{2}\right)^{2}} \sum_{j=M+1}^{N}\left\{x(j)-\sum_{m=1}^{M} a(m) x(j-m)\right\}^{2}
$$

then

$$
\sigma^{2}=\frac{1}{N-M} \sum_{s=M+1}^{N}\left\{x(s)-\sum_{m=1}^{M} a(m) x(s-m)\right\}^{2}
$$

Thus

$\log f\left(x \mid \hat{\sigma}_{M}^{2}, \hat{a}_{M}\right)=-\frac{N-M}{2} \log 2 \pi \hat{\sigma}_{M}^{2}-\frac{N-M}{2}$ 
D. Yule Walker Method ${ }^{3}$

Assuming

$x(0), x(-1), \cdots, x(1-M), x(n+1), \cdots, x(n+M)$

$=0$,

then

$\log f\left(x \mid \sigma^{2}, a_{M}\right)=N \log \left(2 \pi \sigma^{2}\right)^{-\frac{1}{2}}$

$-\frac{1}{2 \sigma^{2}} \sum_{s=1}^{N+M}\left\{x(s)-\sum_{m=1}^{M} a(m) a(s-m)\right\}^{2}$

The Yule Walker equation which allows minimizing equation (11) is as follows,

$C_{M} a_{M}=c_{M}$

where $\mathrm{c}_{\mathrm{M}}$ denotes autocorrelation based covariance matrix and is expressed as follows,

$$
\begin{aligned}
c(k) & =\frac{1}{N} \sum_{s=1}^{N-k} x(s) x(s+k) \\
C_{M} & =\left[\begin{array}{ccccc}
c(0) & c(1) & \cdots & c(M-2) & c(M-1) \\
c(1) & c(0) & c(1) & \cdots & c(M-2) \\
\vdots & \ddots & \ddots & \ddots & \vdots \\
c(M-2) & \cdots & c(1) & c(0) & c(1) \\
c(M-1) & c(M-2) & \cdots & c(1) & c(0)
\end{array}\right] \\
c_{M} & =(c(1), c(2), \cdots, c(M))^{T}
\end{aligned}
$$

Solution of Yule Walker equation (12) can be expressed as $\hat{a}_{M}$, then,

$$
\hat{\sigma}_{M}^{2}=c(0)-c_{M}^{T} \hat{a}_{M}
$$

At this time, the maximum logarithmic function of likelihood is expressed as follows,

$\log f\left(x \mid \hat{\sigma}_{M}^{2}, \hat{a}_{M}\right)=N \log \left(2 \pi \hat{\sigma}_{M}^{2}\right)^{-\frac{1}{2}}-\frac{N}{2}$

\section{E. Time Series of Multi-Variables Prediction}

The aforementioned prediction method can be used for prediction of time series of multi-variables,

$$
\begin{aligned}
& \mathbf{X}_{n}=\left(x_{n}(1), \cdots, x_{n}(k)\right)^{T} \\
& E[\mathbf{U}(n)]=\left[\begin{array}{c}
0 \\
\vdots \\
0
\end{array}\right]
\end{aligned}
$$

http://ja.wikipedia.org/wiki/\%E8\%87\%AA\%E5\%B7\%B1\%E5\%9B\%9E\%E5 $\%$ B8\%B0\%Е7\%A7\%BB\%E5\%8B\%95\%Е5\%B9\%B3\%E5\%9D\%87\%Е3\%83 $\% \mathrm{~A} 2 \% \mathrm{E} 3 \% 83 \% 87 \% \mathrm{E} 3 \% 83 \% \mathrm{AB}$

$$
\begin{aligned}
& E\left[\mathbf{U}(n) \mathbf{U}(n)^{T}\right]=\left[\begin{array}{ccc}
\sigma_{11} & \cdots & \sigma_{1 k} \\
\vdots & \ddots & \vdots \\
\sigma_{k 1} & \cdots & \sigma_{k k}
\end{array}\right]=W \\
& E\left|\mathbf{U}(n) \mathbf{U}(n)^{T}\right|=[0] \quad(n \neq m) \\
& E\left[\mathbf{U}(n) \mathbf{X}(n-m)^{T}\right]=[0] \quad(m \geq 1)
\end{aligned}
$$

Then the expectation is expressed as follows,

$$
\begin{aligned}
& E\left[\mathbf{U}(n) \mathbf{X}(n)^{T}\right] \\
& =E\left[\mathbf{U}(n)\left\{\sum_{m=1}^{M} \mathbf{X}(n-m)^{T} A(m)^{T}+\mathbf{U}(n)^{T}\right\}\right] \\
& =\sum_{m=1}^{M} E\left[\mathbf{U}(n) \mathbf{X}(n-m)^{T}\right] A(m)^{T}+E\left[\mathbf{U}(n) \mathbf{U}(n)^{T}\right] \\
& =E\left[\mathbf{U}(n) \mathbf{U}(n)^{T}\right] \\
& =W
\end{aligned}
$$

On the other hands, autocorrelation is expressed as follows,

$$
\begin{aligned}
& R(m)=E\left[\mathbf{X}(n) \mathbf{X}(n-m)^{T}\right]=\left[\begin{array}{ccc}
r_{11}(m) & \cdots & r_{1 k}(m) \\
\vdots & \ddots & \vdots \\
r_{k 1}(m) & \cdots & r_{k k}
\end{array}\right] \\
& r_{i j}(m)=E\left[x_{i}(n) x_{j}(n-m)\right]
\end{aligned}
$$

There is the following relation between the expectation and the autocorrelation,

$$
\begin{aligned}
& \begin{aligned}
R(-m) & =E\left[\mathbf{X}(n) \mathbf{X}(n+m)^{T}\right] \\
& =E\left[\mathbf{X}(n-m) \mathbf{X}(n)^{T}\right] \\
& =E\left[\mathbf{X}(n) \mathbf{X}(n-m)^{T}\right]^{T} \\
& =R(m)^{T}
\end{aligned} \\
& R(m)=E\left[\left\{\sum_{j-1}^{M} A(j) \mathbf{X}(n-j)+\mathbf{U}(n)\right\} \mathbf{X}_{-}(n-m)^{T}\right] \\
& =\sum_{j=1}^{M} A(j) E\left[\mathbf{X}(n-j) \mathbf{X}(n-m)^{T}\right]+E\left[\mathbf{U}(n) \mathbf{X}(n-m)^{T}\right]
\end{aligned}
$$

Then

$$
\begin{aligned}
& R(0)=\sum_{j=1}^{M} A(j) R(-j)+W \\
& R(m)=\sum_{j=1}^{M} A(j) R(m-j) \quad(m=1,2, \cdots)
\end{aligned}
$$

If the time series of multi variables are stationery, then

$$
\begin{aligned}
R(m) & =E\left[\mathbf{X}(n) \mathbf{X}(n-m)^{T}\right] \\
& =\lim _{N \rightarrow \infty} \frac{1}{N} \sum_{n=1}^{N} \mathbf{X}(n) \mathbf{X}(n-m)^{T}
\end{aligned}
$$

Then covariance matrix is expressed a s follows,

$$
\begin{aligned}
& C(m)=\frac{1}{N} \sum_{n=m+1}^{N} \mathbf{X}(n) \mathbf{X}(n-m)^{T} \\
& C_{i j}(m)=\frac{1}{N} \sum_{n=m+1}^{N} x_{i}(n) x_{j}(n-m)^{T}
\end{aligned}
$$


Thus autoregressive model of $\hat{A}(j)$ can be determined as follows,

$$
\begin{aligned}
& C(m)=\sum_{j=1}^{M} A(j) C(m-j) \\
& \hat{V}_{m}=C(0)-\sum_{j=1}^{M} \hat{A}(j) C(-j)
\end{aligned}
$$

In order to determine the most appropriate order (or the number of possible predictions) of the autoregressive model, Akaike's Information Criteria: AIC is introduced,

$\mathrm{AIC}=-2 \mathrm{~L}+2 \mathrm{P}$

where $\mathrm{L}$ denotes the maximum logarithmic function of likelihood while $\mathrm{P}$ denotes the number of adjustable parameters. Therefore,

$$
A I C(m)=N k \log 2 \pi+N \log \left|\hat{V}_{m}\right|+N k+2 m k^{2}+k(k+1)
$$

where

$$
\begin{gathered}
L=-\frac{N}{2}\left(k \log 2 \pi+\log \left|\hat{V}_{m}\right|+k\right) \\
\mathrm{P}=m k^{2}+\frac{k(k+1)}{2}
\end{gathered}
$$

Remove the terms which are not related to the order of $\mathrm{m}$,

$$
A I C^{*}=N \log \left|\hat{V}_{m}\right|+2 m k^{2}
$$

is used to be used usually.

\section{EXPERIEMNTS}

\section{A. Time Series of Imagery Data Used}

Figure 1 shows experimental time series of imagery data of Visible/Infrared Spin Scan-Radiometer: VISSR onboard Geostationary Meteorological Satellite: GMS acquired time series of imagery data.

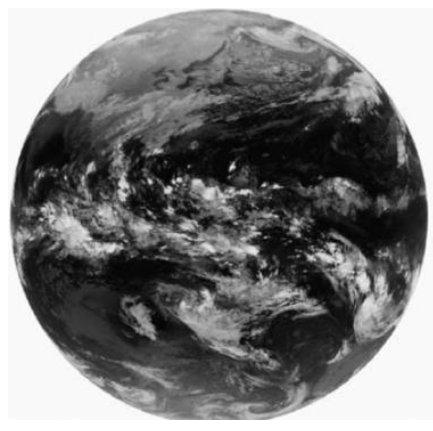

(a)Infrared 1

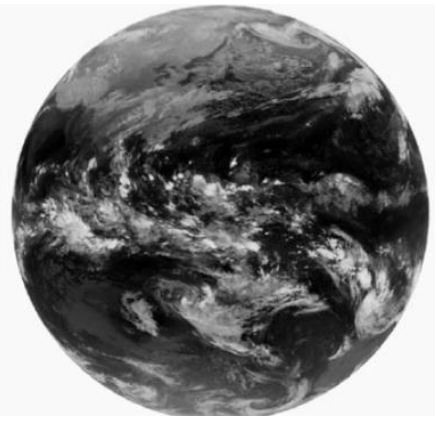

(b)Infrared 2
Figure 1 VISSR/GMS of time series of imagery data which was acquired at 16:25 on 12 December 2000.

VISSR/GMS has four channels, 0.55-0.9, 10.5-11.5, 11.512.5, 6.5-7.0 $\mu \mathrm{m}$, Infrared 1 denotes $10.5-11.5$ while infrared 2 denotes $11.5-12.5$, respectively.
VISSR/GMS imagery data can be acquired every one hour. From the VISSR/GMS images, 100 by 100 pixels of small portion of images are extracted. Portion of images of the same area of South East China sea area are extracted as shown in Figure 2.

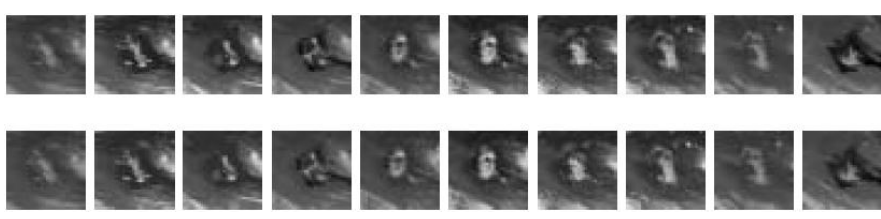

Figure 2 Time series of VISSR/GMS images of infrared 1(Top) and 2(Bottom) channels of data used for experiments

B. $A I C$

41 of time series of VISSR/GMS infrared 1 and 2 channels of imagery data are used for prediction. AIC is estimated with the experimental data in the real world space and in the eigen space. AIC order in real space is $3,5,10$ while that in eigen space is $2,5,10$. Therefore, 10 of time series of imagery data of VISSR/GMS are tried to predict. Although autoregressive model parameters are estimated with 31 of imagery data, the imagery data which are acquired 10 hour later is available

AIC is calculated for each order using covariance matrix in accordance with equation (13). The results are shown in Table 1.

TABLE I. AIC CALCULATED WITH COVARIANCE METRIX

\begin{tabular}{|l|r|r|r|}
\hline \multicolumn{2}{|c|}{} & Eigen space & Autoregressive \\
\hline \multirow{4}{*}{ IR1 } & 3 & 132.9 & 169.5 \\
\cline { 2 - 4 } & 5 & 139.3 & 174.3 \\
\cline { 2 - 4 } & 10 & 160.2 & 200.1 \\
\hline \multirow{3}{*}{ IR2 } & 3 & 119.3 & 205.3 \\
\cline { 2 - 4 } & 5 & 127.8 & 210.8 \\
\cline { 2 - 4 } & 10 & 143.6 & 228.4 \\
\hline & 3 & 265.7 & 374.9 \\
\cline { 2 - 4 } & 5 & 252.1 & 361.4 \\
\cline { 2 - 4 } & 10 & 265.6 & 389.4 \\
\hline
\end{tabular}

In the table, IR1 and IR2 denotes infrared 1 and 2 VISSR/GMS channels of imagery data while two variables means the case of prediction by using IR1 and IR2 together. AIC are different between eigen space and real space (the conventional autoregressive model). Therefore, both AICs are calculated. In general, smaller AIC implies better prediction accuracy.

\section{RMS Error}

It is possible to evaluate Root Mean Square: RMS error between the ten of original images and the predicted images of the corresponding time. The estimated RMS errors are shown in Figure 3. Figure 3 shows RMS error for the single variable cases.

Figure 4 shows the experimental results of RMS error for the two variables cases. 


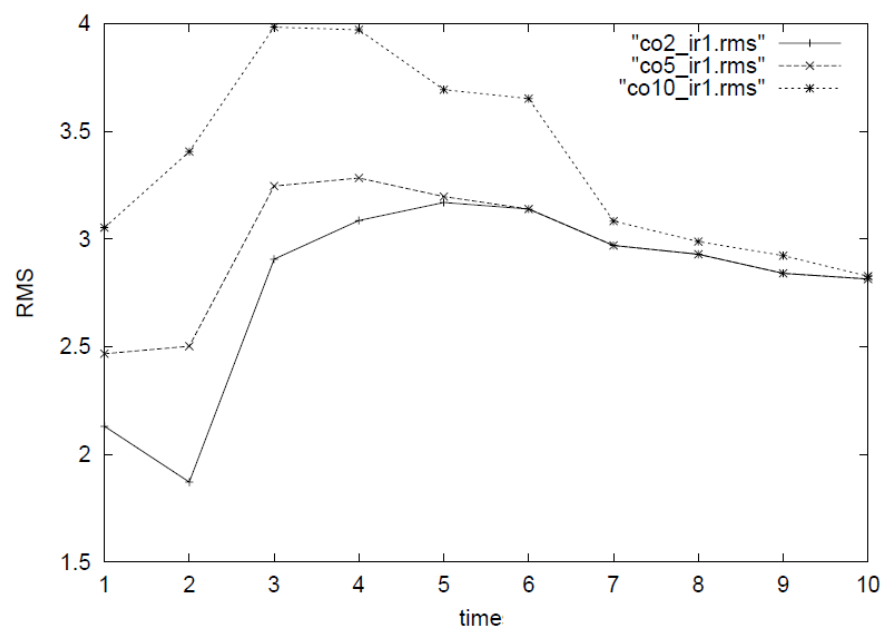

(a)Eigen space for IR1

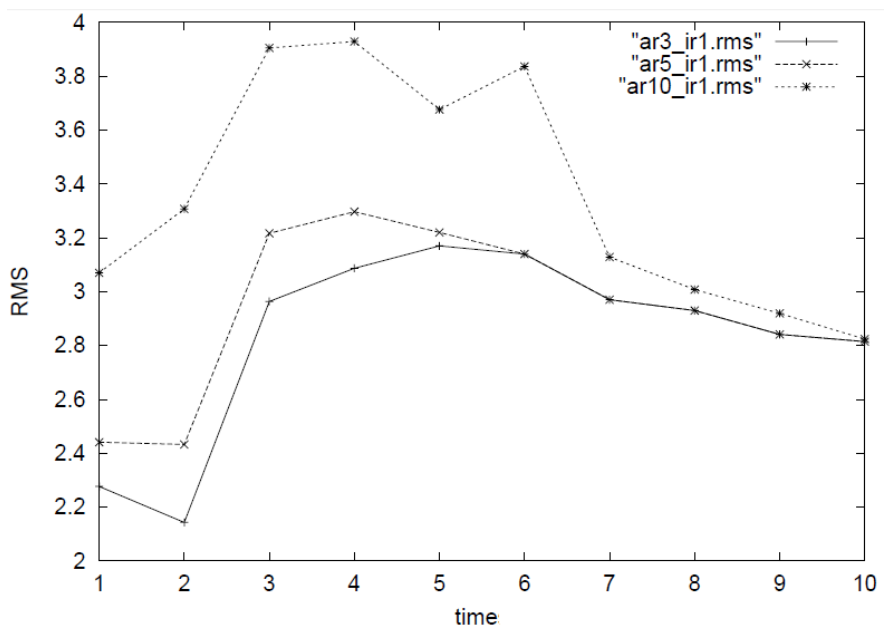

(b)Real space for IR1

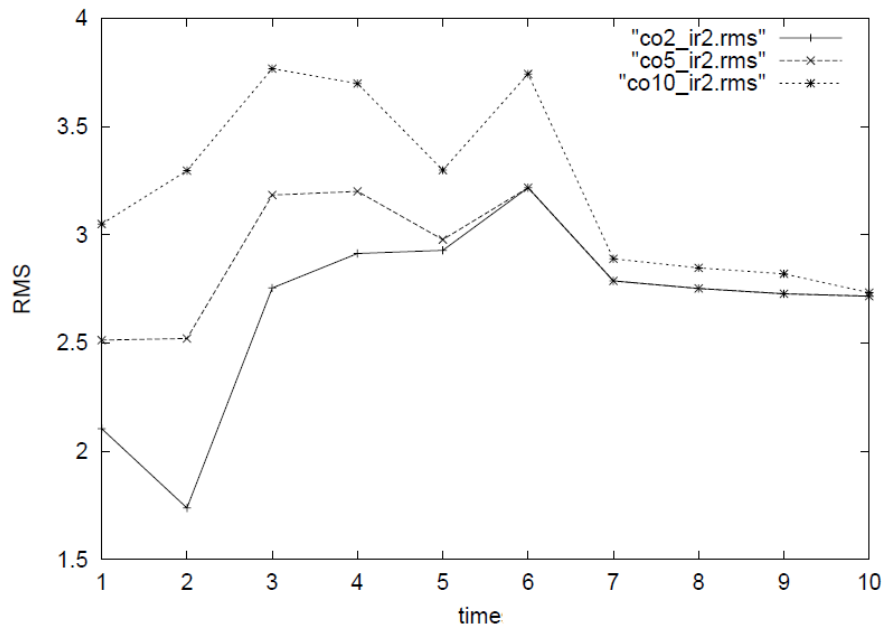

(c)Eigen space for IR2

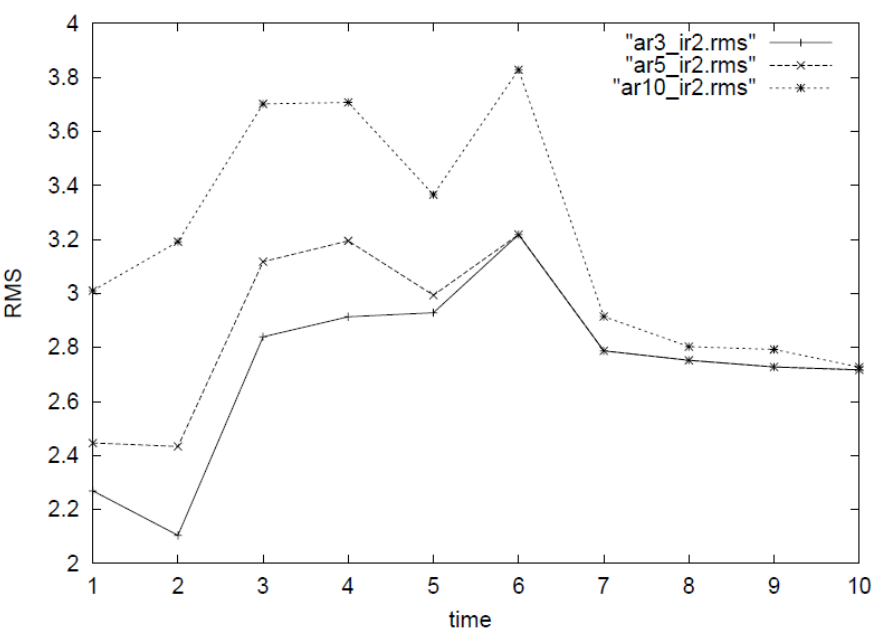

(d)Real space for IR2

Figure 3 RMS error evaluated with VISSR/GMS time series of images for 10 unit time later data predictions based on the autoregressive models represented in real and eigen spaces for single variable cases.

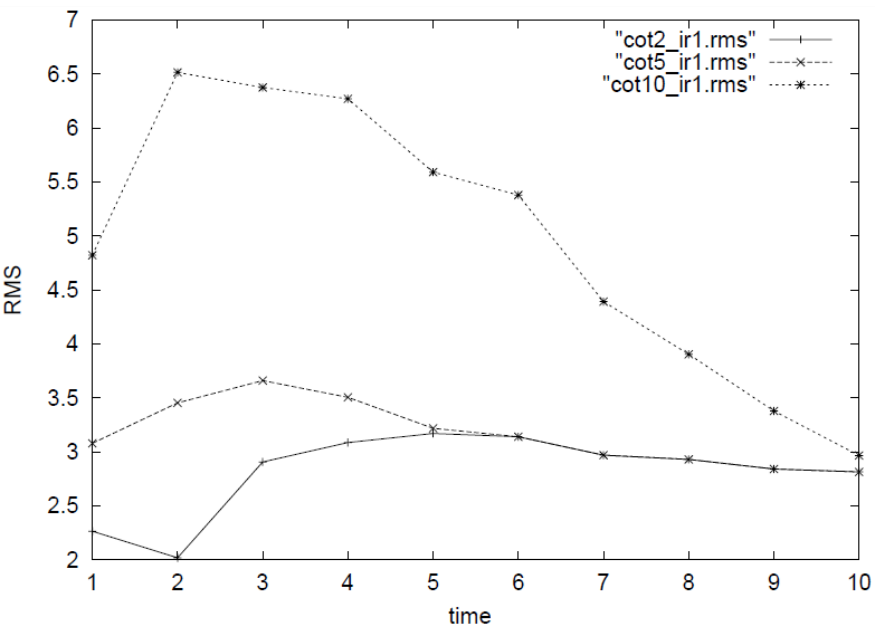

(a)Eigen space for IR1

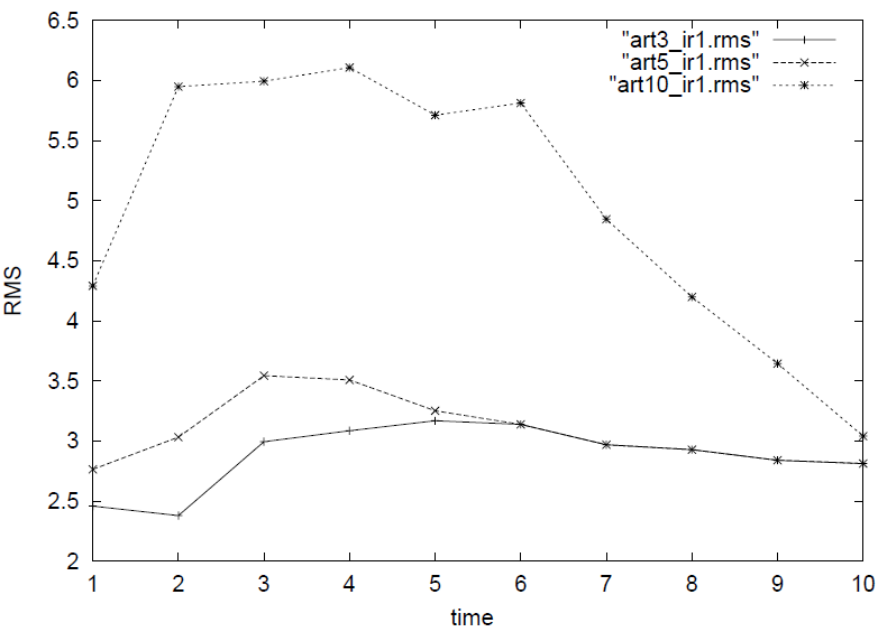

(b)Real space for IR2 


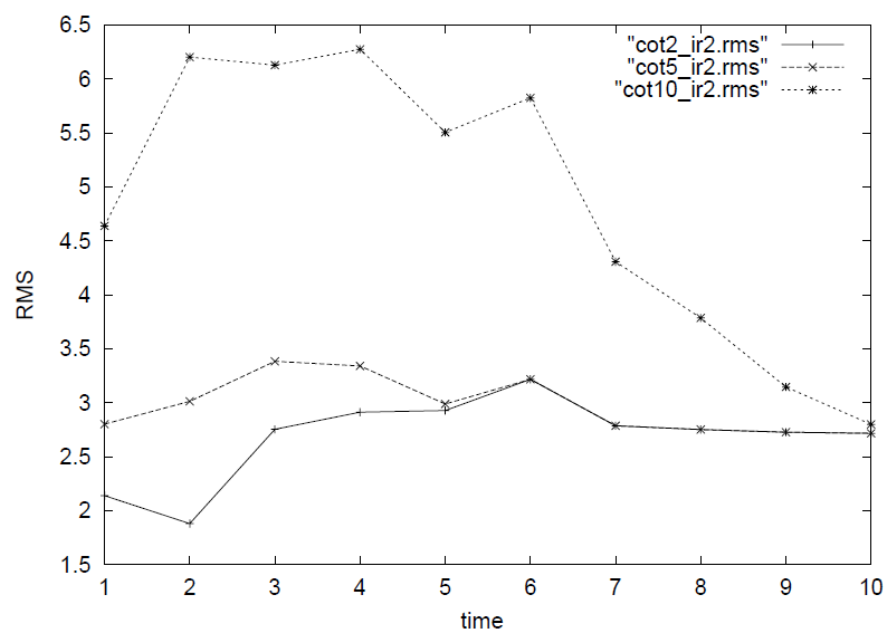

(c)Eigen space for IR1

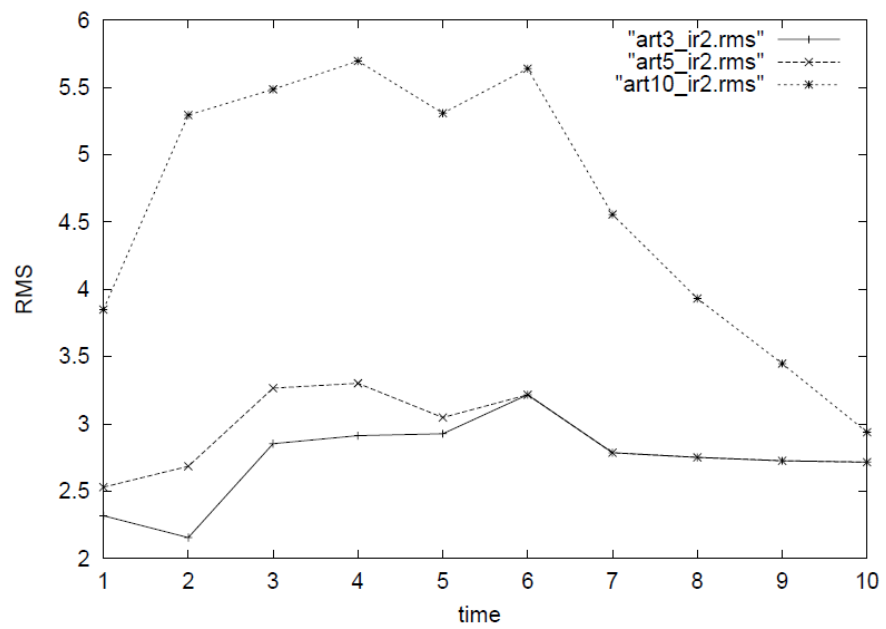

(d)Real space for IR2

Figure 4 RMS error evaluated with VISSR/GMS time series of images for 10 unit time later data predictions based on the autoregressive models represented in real and eigen spaces for two variable cases.

\section{Original and Predicted Images and RMS Error for IRI}

Figure 5 (b) shows the predicted images for the conventional real space based method while Figure 5 (c) shows those for the proposed eigen space based method.
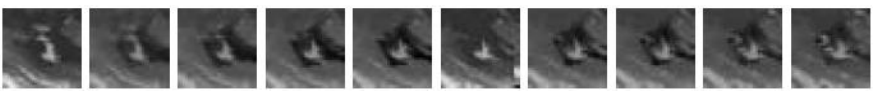

(a)Original images
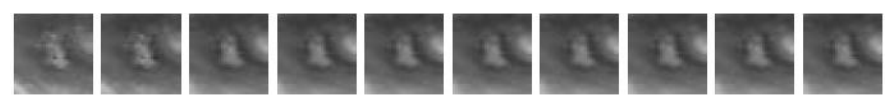

(b)Real space based
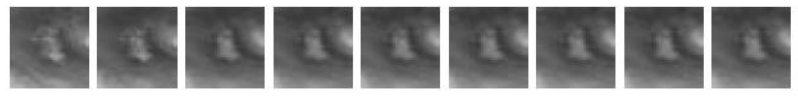

(c)Eigen space based

Figure 5 Original and predicted images based on the conventional real space and the proposed eigen space based methods
Figure 5 (a) shows the 10 hours of time series of the original portion of VISSR/GMS images while Figure 5 (b) shows the predicted images at the corresponding time for Figure 5 (a). Also Figure 5 (c) shows the predicted images at the corresponding time.

Prediction performances in terms of RMS error between the original and the predicted images for the conventional and the proposed methods are summarized in Table 2. Table 2 also shows improvement of the proposed method in comparison to the conventional real space based method. The RMS errors for the first to third images show some improvements of prediction accuracy in comparison between the conventional and the proposed method while the RMS errors for fourth or later images show no improvement. Then the proposed prediction method is effective for first to third time periods and is not effective for further time period.

As shown in Figure 6, RMS errors for real space (ar3_ir1.rms) and eigen space (co2_ir1.rms) are getting close each other

TABLE II. RMS ERROR BETWEEN THE ORIGINAL AND THE PREDICTED IMAGES BASED ON REAL SPACE AND EIGEN SPACE BASED METHODS FOR IR1

\begin{tabular}{|r|r|r|r|}
\hline n-th image & Eigen space & Real space & Prediction improvement \\
\hline 1 & 2.131 & 2.276 & 6.4 \\
\hline 2 & 1.871 & 2.143 & 12.7 \\
\hline 3 & 2.907 & 2.963 & 1.9 \\
\hline 4 & 3.086 & 3.086 & 0 \\
\hline 5 & 3.17 & 3.17 & 0 \\
\hline 6 & 3.139 & 3.139 & 0 \\
\hline 7 & 2.97 & 2.97 & 0 \\
\hline 8 & 2.929 & 2.929 & 0 \\
\hline 9 & 2.841 & 2.841 & 0 \\
\hline 10 & 2.814 & 2.814 & 0 \\
\hline & & & 0 \\
\hline
\end{tabular}

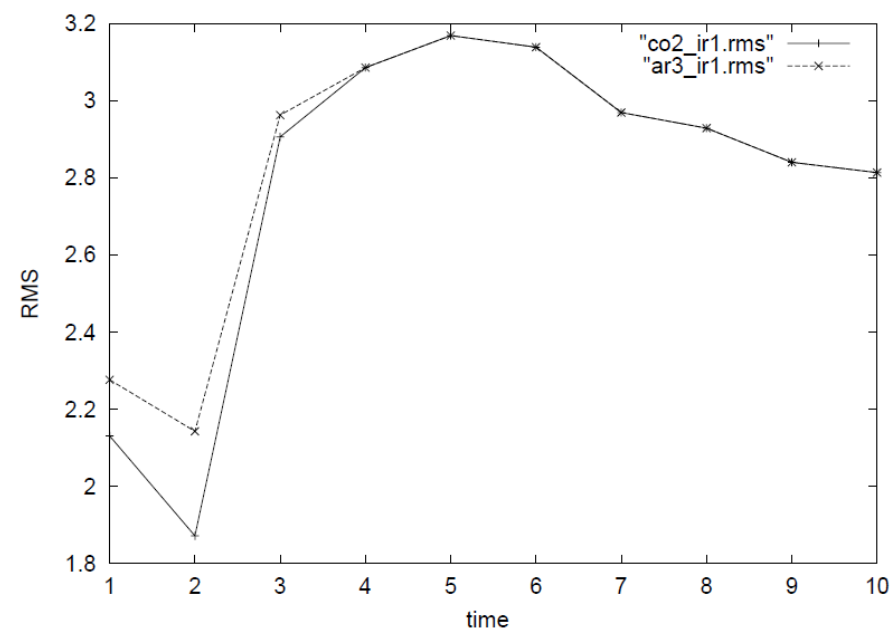

Figure 6 RMS errors for real space (ar3_ir1.rms) and eigen space (co2_ir1.rms) 


\section{E. Original and Predicted Images and RMS Error for IR2}

Figure 7 (b) shows the predicted images for the conventional real space based method while Figure 7 (c) shows those for the proposed eigen space based method.
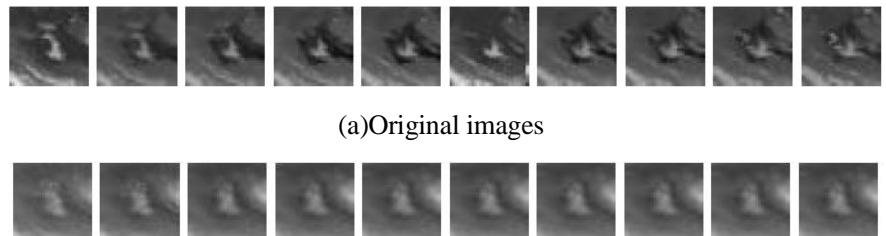

(a)Original images
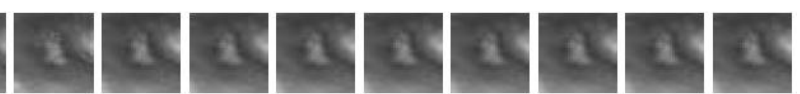

(b)Real space based
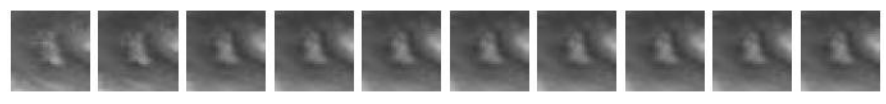

(c)Eigen space based

Figure 7 Original and predicted images based on the conventional real space and the proposed eigen space based methods

Figure 7 (a) shows the 10 hours of time series of the original portion of VISSR/GMS images while Figure 7 (b) shows the predicted images at the corresponding time for Figure 7 (a). Also Figure 7 (c) shows the predicted images at the corresponding time.

Prediction performances in terms of RMS error between the original and the predicted images for the conventional and the proposed methods are summarized in Table 3. Table 3 also shows improvement of the proposed method in comparison to the conventional real space based method. The RMS errors for the first to third images show some improvements of prediction accuracy in comparison between the conventional and the proposed method while the RMS errors for fourth or later images show no improvement. Then the proposed prediction method is effective for first to third time periods and is not effective for further time period.

As shown in Figure 8, RMS errors for real space (ar3_ir2.rms) and eigen space (co2_ir2.rms) are getting close each other

TABLE III. RMS ERROR BETWEEN THE ORIGINAL AND THE PREDICTED IMAGES BASED ON REAL SPACE AND EIGEN SPACE BASED METHODS FOR IR2

\begin{tabular}{|r|r|r|r|}
\hline n-th image & Eigen space & Real space & Prediction improvement \\
\hline 1 & 2.104 & 2.269 & 7.3 \\
\hline 2 & 1.738 & 2.104 & 17.4 \\
\hline 3 & 2.754 & 2.839 & 3 \\
\hline 4 & 2.913 & 2.913 & 0 \\
\hline 5 & 2.928 & 2.928 & 0 \\
\hline 6 & 3.217 & 3.217 & 0 \\
\hline 7 & 2.787 & 2.787 & 0 \\
\hline 8 & 2.752 & 2.752 & 0 \\
\hline 9 & 2.727 & 2.727 & 0 \\
\hline 10 & 2.717 & 2.717 & 0 \\
\hline
\end{tabular}

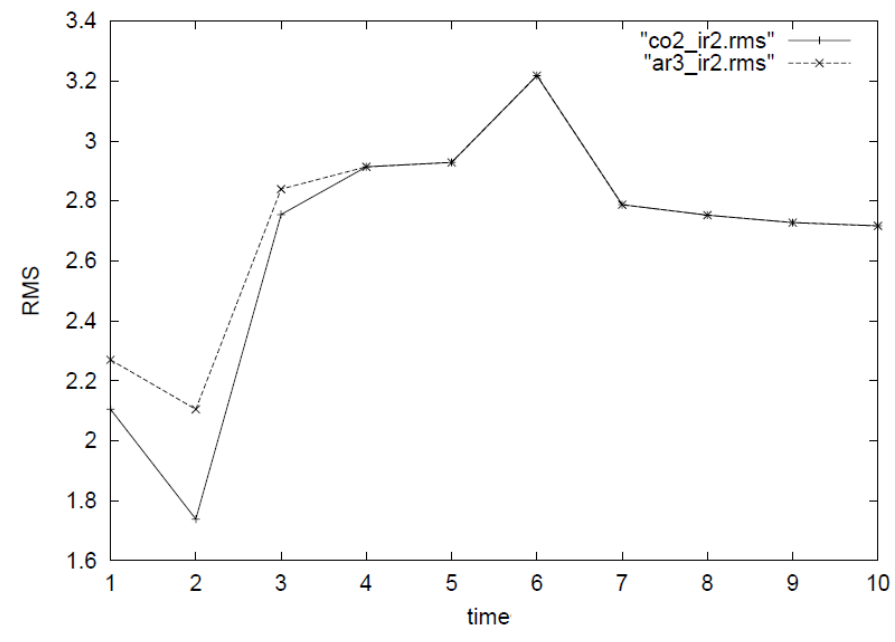

Figure 8 RMS errors for real space (ar3_ir2.rms) and eigen space (co2_ir12.rms)

\section{F. Original and Predicted Images and RMS Error for Two Variables Cases}

Figure 9 (a) shows the predicted images for the conventional real space based method while Figure 9 (b) shows those for the proposed eigen space based method. Figure 9 (a) shows the predicted images at the corresponding time for Figure 5 (a). Also Figure 9 (b) shows the predicted images at the corresponding time.
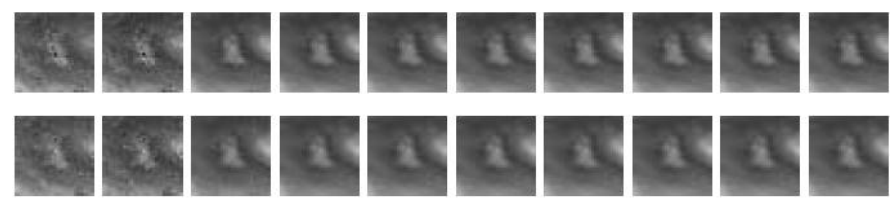

(a)Real space based
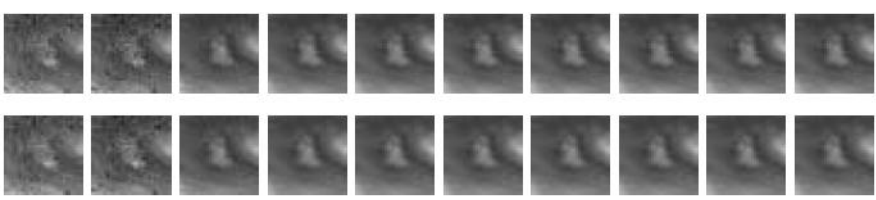

(b)Eigen space based

Figure 9 Original and predicted images based on the conventional real space and the proposed eigen space based methods

Prediction performances in terms of RMS error between the original and the predicted images for the conventional and the proposed methods are summarized in Table 4 . Table 4 also shows improvement of the proposed method in comparison to the conventional real space based method. The RMS errors for the first to third images show some improvements of prediction accuracy in comparison between the conventional and the proposed method while the RMS errors for fourth or later images show no improvement. Then the proposed prediction method is effective for first to third time periods and is not effective for further time period. As shown in Figure 10, RMS errors for real space (ar3_ir2.rms) and eigen space (co2_ir2.rms) are getting close each other. This implies that the proposed time series analysis in eigen space is effective for the prediction within three recent unit times and is not effective for no further unit time. 
TABLE IV. RMS ERROR BETWEEN THE ORIGINAL AND THE PREDICTED IMAGES BASED ON REAL SPACE AND EIGEN SPACE BASED METHODS FOR TWO VARIABLES CASES

\begin{tabular}{|r|r|r|l|l|l|r|}
\cline { 2 - 7 } \multicolumn{1}{c|}{} & \multicolumn{1}{l|}{ IR1 } & \multicolumn{1}{l|}{ IR2 } \\
\hline \begin{tabular}{r|r|r|r|r|} 
n-th \\
future
\end{tabular} & $\begin{array}{l}\text { Eigen } \\
\text { space }\end{array}$ & $\begin{array}{l}\text { Real } \\
\text { space }\end{array}$ & $\begin{array}{l}\text { Prediction } \\
\text { improvement }\end{array}$ & $\begin{array}{l}\text { Eigen } \\
\text { space }\end{array}$ & $\begin{array}{l}\text { Real } \\
\text { space }\end{array}$ & $\begin{array}{l}\text { Prediction } \\
\text { improvement }\end{array}$ \\
\hline 1 & 2.264 & 2.46 & 8 & 2.139 & 2.319 & 7.8 \\
\hline 2 & 2.018 & 2.381 & 15.3 & 1.88 & 2.157 & 12.9 \\
\hline 3 & 2.907 & 2.996 & 3 & 2.754 & 2.854 & 3.6 \\
\hline 4 & 3.086 & 3.086 & 0 & 2.913 & 2.913 & 0 \\
\hline 5 & 3.17 & 3.17 & 0 & 2.928 & 2.928 & 0 \\
\hline 6 & 3.139 & 3.139 & 0 & 3.217 & 3.217 & 0 \\
\hline 7 & 2.97 & 2.97 & 0 & 2.787 & 2.787 & 0 \\
\hline 8 & 2.929 & 2.929 & 0 & 2.752 & 2.752 & 0 \\
\hline 9 & 2.841 & 2.841 & 0 & 2.727 & 2.727 & 0 \\
\hline 10 & 2.814 & 2.814 & 0 & 2.717 & 2.717 & 0 \\
\hline
\end{tabular}

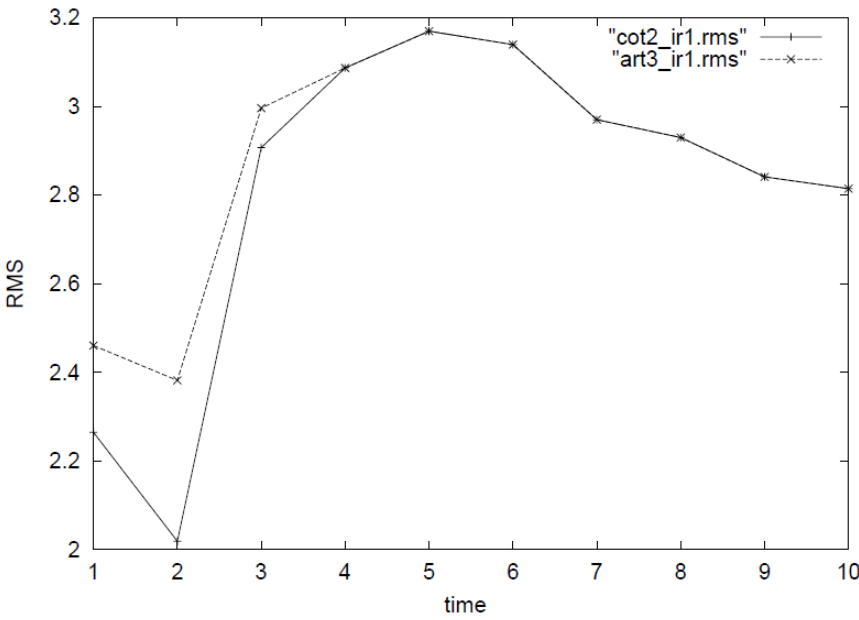

(a)IR1

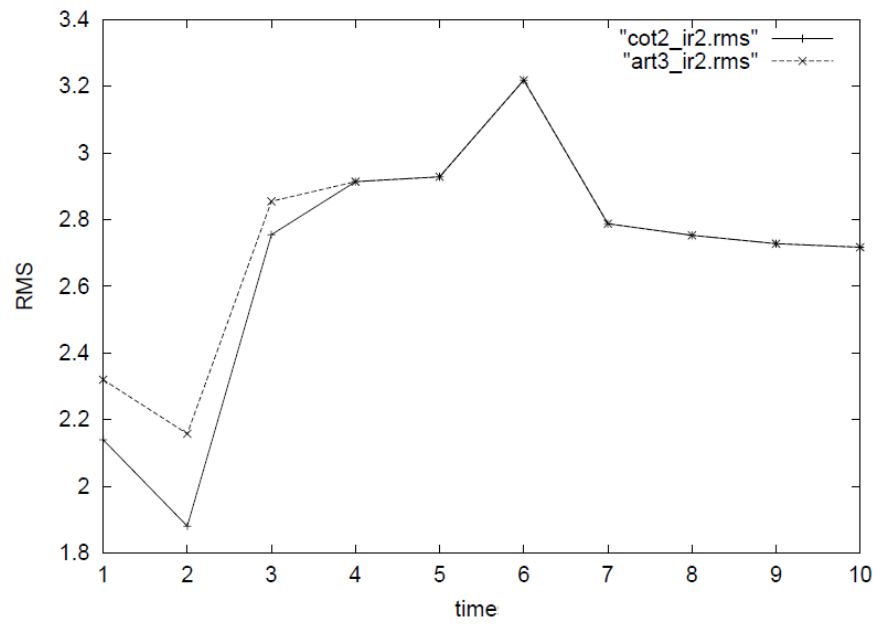

(b)IR2

Figure 10 RMS errors for real space (ar3_ir2.rms) and eigen space (co2 ir12.rms)

\section{CONCLUSION}

Prediction method for time series of imagery data on eigen space is proposed. Although the conventional prediction method is defined on the real world space and time domains, the proposed method is defined on eigen space. Prediction accuracy of the proposed method is supposed to be superior to the conventional methods. Through experiments with time series of satellite imagery data, validity of the proposed method is confirmed. The proposed time series analysis in eigen space is effective for the prediction within three recent unit times and is not effective for no further unit time

\section{ACKNOWLEDGMENT}

The author would like to tank Dr. Yasunori Terayama and Mr. Masaya Ishida for their effort to conduct experiments.

\section{REFERENCES}

[1] Bloomfield, P., Fourier analysis of time series: An introduction. New York: Wiley, 1976.

[2] Shumway, R. H., Applied statistical time series analysis. Englewood Cliffs, NJ: Prentice Hall, 1988.

[3] Gershenfeld, N., The nature of mathematical modeling. p.205-08, 1999.

[4] Boashash, B. (ed.), Time-Frequency Signal Analysis and Processing: A Comprehensive Reference, Elsevier Science, Oxford, 2003 ISBN ISBN 0-08-044335-4, 2003.

[5] Nikolić D, Muresan RC, Feng W, Singer W, Scaled correlation analysis: a better way to compute a cross-correlogram. European Journal of Neuroscience, pp. 1-21, doi:10.1111/j.1460-9568.2011.07987.x, 2012. http://www.danko-nikolic.com/wp-content/uploads/2012/03/Scaledcorrelation-analysis.pdf

[6] K. Arai, Fundamental Theory and Algorithms on Image Processing, Gakujutsu-Tosho Publishing Co. Ltd., 1995.

\section{AUTHORS PROFILE}

Kohei Arai, He received BS, MS and PhD degrees in 1972, 1974 and 1982, respectively. He was with The Institute for Industrial Science, and Technology of the University of Tokyo from 1974 to 1978 also was with National Space Development Agency of Japan (current JAXA) from 1979 to 1990. During from 1985 to 1987, he was with Canada Centre for Remote Sensing as a Post Doctoral Fellow of National Science and Engineering Research Council of Canada. He was appointed professor at Department of Information Science, Saga University in 1990. He was appointed councilor for the Aeronautics and Space related to the Technology Committee of the Ministry of Science and Technology during from 1998 to 2000. He was also appointed councilor of Saga University from 2002 and 2003 followed by an executive councilor of the Remote Sensing Society of Japan for 2003 to 2005. He is an adjunct professor of University of Arizona, USA since 1998. He also was appointed vice chairman of the Commission "A" of ICSU/COSPAR in 2008. He wrote 30 books and published 332 journal papers 